\title{
The importance of experience in using cervical pessary for prevention of preterm birth
}

\begin{abstract}
Cervical pessary is a new treatment for preterm birth and have obtained a lot of space in clinical practice because its real efficacy, which unconsidered the bias produced by some research.

There is a lack of evidence about cervical pessary in many aspects and only those who handle this device every day can note and call for attention. Experience is the central point about cervical pessary in this article, and important centers with training and expertise must be taken the front of this issue, because low experience probably is one of the causes of contradictory results in literature. In fact, we have a lot of aspects that need attention and never were treated because always the efficacy is questioned and do not open space for other important aspects.

We have a lot of o issues to discuss during this article such as the indication of a cervical pessary, the needs of guidelines about pessary, including a learning curve of pessary treatment, the relevant aspects about insertion of the device, its association with other medications, needs for hospitalization, which is the best management for the vaginal discharge pessary related, how to handle the pelvic pain after insertion, the urinary symptoms, the obstipation, and the inhibition of preterm labor during pessary treatment, and finally, what are the relevant aspects about the withdrawal of cervical pessary?
\end{abstract}

Volume 12 Issue 2 - 202I

\author{
Marcelo Santucci Franca,Alan Roberto \\ Hatanaka,Antonio Fernandes Moron \\ Department of Obstetrics, Federal University of Sao Paulo - \\ UNIFESP/EPM, Brazil
}

Correspondence: Dr. Marcelo Santucci Franca, MD, Senior Medical Researcher from Sector of Prediction and Prevention of Preterm Birth, Department of Obstetrics, Federal University of Sao Paulo - UNIFESP/EPM, Brazil,

Email marcelosantucci.franca@gmail.com

Received: March 15, 202 I | Published: April 01, 2021

\section{Introduction}

Since the publication of Goya et al. in 2012 concerning the use of cervical pessary (CP) for the prevention of preterm birth, several studies have been published containing several conflicting results, ${ }^{1-5}$ including some metanalyses. ${ }^{6-10}$ These unexpected discrepancies in outcomes can be explained by several reasons, such as distinct selection criteria, ethnic and cultural particularities. One point that is not always discussed is the lack of training on how to handle the CP.

The studies related to short cervix were conducted in multicenter trials, and the outcome was evaluated, or the influence of a particular medicine was tested. However, studies with these purposes are different when it comes to medical procedures or surgeries.

Research using progesterone in women with short cervix does not demand experience or training of the medical staff. Differently, studies with surgical interventions need training and uniformization of the technique. The team involved in $\mathrm{CP}$ research should be familiar with the device, procedure, indication and also understand the mechanisms involved to achieve the results, side effects, techniques of insertion and removal, and its expected performance. We believe that without a fully trained team, it is very hard to obtain adequate results with this procedure.

It is plausible to understand that if an Obstetrician that has not already used CP receives a woman with complaints of massive vaginal discharge, difficulty in evacuating, and pain, have the intention of removing the device to return to their previous known status.

During the last ten years, we are using cervical pessary in preterm birth prevention in our clinical practice. In the beginning, some patients that we inserted pessary were counseled to remove the device without any medical reason and being reported only as removal at the patient's request. We gradually realized that vaginal discharge was a physiological reaction and the difficulty in evacuating and the pain was transient, and we started to have the devices removed very rarely.

There is a study that related that several patients removed the CP with or without a proper medical reason. ${ }^{1}$ However, we believe that there are two medical reasons accepted for the removal of the pessary before 34 weeks: PPROM and preterm labor that efforts to inhibition failed. And these aspects are very rare and represent no more than $7 \%$ of the cases of preterm birth. ${ }^{11}$ Therefore, a study with a large number of pessary removal, even with medical indication, could be the reason for conflicting results.

Different from a trial with medicine, the pessary removal can be the reason itself for preterm delivery. If we consider intention-to-treat in drug treatment, the withdrawal after the use of the drug just returns to the initial status of the patient, to the time of inclusion of the trial. In addition, the period of treatment increases the chance that the event will not occur, always improving the outcome, i.e., if the compliance increases, better performance is expected.

On the other hand, in studies with surgery or device performance, this rationale (intention-to-treat) cannot be applied in most cases, because the interruption of the treatment could start a new event, and the period in use could not be enough to help the patient. The removal of CP can be more traumatic than its insertion and can trigger labor. Usually, the removal of $\mathrm{CP}$ can be traumatic and can separate the cervix from the amniotic membrane, similar to Hamilton's maneuver, and can cause uterine contractions. Thus, the over-indication of pessary removal should be avoided, because the withdrawal itself can be a cause of more preterm deliveries. And, finally, intention-totreat must be avoided in cervical pessaries studies, because removing the device precociously could be worse than never using it. In our opinion, if the patient requests removal without a medical reason, it must be excluded from the trial. 
Using our large experience in $\mathrm{CP}$ we can observe that the two aspects that determine the best outcome are the sonographic visualization of the pessary involving completely the cervix and the training of the entire staff to handle the $\mathrm{CP}$ after the insertion.

The presentation of figures about preterm deliveries, in our tertiary hospital, for the Internal Pharmacologic Committee was auspicious. We could show that during the first four years of the study when treatment was not systematic, we had 23 cases of preterm deliveries before 34 weeks in a cohort of 237 patients (excluded all mechanic treatments - pessary and cerclage). In the middle of 2015, we started a randomized clinical trial associated to systematic treatment for short cervix (P5 Study) ${ }^{12,13}$ and, in a cohort of 287 patients excluding all the $\mathrm{CP}$ and cerclage, we had only 13 preterm deliveries $<34$ weeks, a decrease from $9.7 \%$ to $4.5 \%$. Even the number of preterm births $<37$ weeks decreased from 44 to 26 , a reduction from $18.5 \%$ to $9.0 \%$. We had a budget during this trial of around US $\$ 10,000.00$, and the cost of a single preterm infant has on average this value for the hospital. ${ }^{14,15}$ After that presentation, the hospital included CP in the non-standard medication of our pharmacy and supported all of our cases and research with this device, based on just the economic aspect.

Some information about cervical pessary has been already published for new users, ${ }^{16-18}$ but many other aspects need to be pointed out about this subject.

\section{General aspects}

It is necessary a learning curve including the number of pessary insertions in a center, which may be useful for societies to develop new guidelines for good clinical practice and continuous medical education. This guideline must contain a minimal number of insertions to the participation of any study center of a CP to offer this treatment as regular therapeutics. Additionally, the guideline must be evaluated and certified by an external audit performed by specialized professionals and by their respective medical societies.

\section{Is the short cervix the unique aspect to consider before treating a patient with CP?}

The cervical measurement $<25 \mathrm{~mm}$ is the main reason to treat the pregnant woman with a $\mathrm{CP}$ due to its higher chance to have a preterm birth $<34$ weeks. ${ }^{19}$ However, other parameters should be considered according to clinical history, such as maternal characteristics, obstetrical history, time of the diagnosis of short cervix, presence of funneling and sludge, and availability of therapeutic methods. Apparently, logistic regression pointed out some population which can present more effectiveness in the treatment with $\mathrm{CP}$, it is the singleton pregnancy, primigravida, with cervical length $<25 \mathrm{~mm} .{ }^{13}$

\section{What are possible indications for pessary use?}

Singleton pregnancies with cervical length $<\mathbf{2 5 m m}$ : The short cervix $<25 \mathrm{~mm}$ is the regular indication for CP usage, and it represents the most important subgroup treated with this device. It is the $2^{\text {nd }}$ Trimester anomaly scan $\left(18^{\text {th }}\right.$ and $24^{\text {th }}$ week $)$ the best period to offer TVU and screen short cervix with a single exam. ${ }^{20}$

\section{Short cervix in twin gestation}

According to ACOG Practice Bulletin, twin pregnancy has 35.3 weeks as the mean gestational age at birth, ${ }^{21}$ making prematurity part of twin gestation outcome, moreover the association between twin pregnancy and short cervix $<25 \mathrm{~mm}$, regularly produces extremely preterm birth (mean $<30$ weeks). ${ }^{22}$ There is some evidence that twins with short cervix can benefit from $\mathrm{CP}^{23-25}$ but it is still a controversial issue. ${ }^{26}$ There is some evidence that the use of $\mathrm{CP}$ in twin pregnancy with a short cervix may produce a similar outcome of low-risk twin gestation. ${ }^{27}$

\section{Cervical amputation or large loop excision}

This patient starts the pregnancy with a short cervix and is a potential group to benefit from CP. In our protocols, it is possible to consider also cervical cerclage for this subgroup during the $12^{\text {th }}$ week..$^{28}$ If the patient starts the prenatal care later than $1^{\text {st }}$ trimester, the $\mathrm{CP}$ can be considered if a short cervix $<25 \mathrm{~mm}$ is detected in the middle gestation. ${ }^{16}$

\section{Is the association with progesterone, or antibiotics, or probiotics important?}

In our research studies, the association of $\mathrm{CP}$ and progesterone is mandatory, because until this moment vaginal micronized progesterone is the gold standard treatment for short cervix, and must be associated with $\mathrm{CP}$ in all cases. ${ }^{29}$ The use of antibiotics is recommended in case of amniotic fluid sludge associated with a short cervix and, during the first week after pessary insertion, antibiotic treatment regularly is used per protocol..$^{30}$ Besides the studies with vaginal microbiome pointing out that Lactobacillus inners could be responsible for a higher incidence of preterm delivery, ${ }^{31}$ there is no evidence so far that probiotics can be helpful for patients with a short cervix, cervical pessary, or even to prevent preterm birth. ${ }^{32}$

\section{Does it have any indication for hospitalization?}

Patients with cervical pessaries, in general, do not need to be hospitalized, except for some particular aspects:

1) Uterine cervical length $<15 \mathrm{~mm}$ associated with amniotic fluid sludge which can benefit from the use of intravenous antibiotics; $; 0,33$

2) Membrane protrusion through the pessary, the association of cerclage with cervical pessary could be considered; ${ }^{34}$

3) Membrane protrusion through the pessary, Trendelenburg's position could be considered in this severe risk situation. ${ }^{16}$

\section{Technical aspects}

How does the technique assure that the pessary is in the highest possible position inside the vagina if the small orifice is pointing out to the uterus? Is the OS in the middle of the small orifice of the pessary? Which is the TVU frequency needed to evaluate CP after the insertion?

The insertion is performed with the patient in the gynecologic position. The procedure starts with a speculum exam, followed by a two-digital vaginal touch. The first exam has the intention to recognize any pathological discharge and indicate vaginal cultures or identify the membrane protrusion, or any cervical lesion which could be a contraindication for $\mathrm{CP}$ insertion. The second exam is executed by the doctor who is responsible for pessary insertion in order to have a tridimensional view of the cervix, and mentally create the $\mathrm{CP}$ position before the insertion. CP must be introduced inside the vagina folded in the middle, and it will open inside the vagina. The direction of the insertion is the posterior fornix, and the middle of $\mathrm{CP}$ must involve the entire cervix. The small orifice of the pessary needs to point upwards, close to the uterus, and the large diameter of $\mathrm{CP}$ must be in touch with the vaginal posterior wall. A soft pressure can be executed to fix the pessary at the highest position in the vagina. The cervix must be entirely involved by pessary and OS must be inside of the small 
diameter of $\mathrm{CP}$, thus the utero-cervical angle can be modified and CP can be effective (33). Immediately after insertion, another TVU must be executed to evaluate if the cervical gland area is in the middle of the orifice of the CP (Figure 1). ${ }^{36}$



Figure I TVU presenting a CP correctly inserted involving the entire cervix, with cervical gland area (CGA) in the middle of the small orifice of the pessary.

A transvaginal ultrasound should be performed one week after the pessary insertion to make sure that the pessary is in the right position and to identify eventual funneling, membrane protrusion, and decreasing the cervical length during pregnancy. ${ }^{37}$ We had a few patients with CP spontaneously out of the position, but all of them occurred during this first week after insertion. In this appointment, it is important to discuss some concerns and doubts related to cervical pessary and also to inquire the patient about the presence of any pelvic discomfort, obstipation, and discharge. We suggest a new TVU during regular appointments of prenatal care until 37 weeks.

\section{Discharge management}

\section{When and how is it necessary to collect vaginal cultures? When is the treatment necessary?}

There is strong evidence that $\mathrm{CP}$ increases vaginal discharge. On the other hand, there is no evidence that the discharge in cervical pessary patients is associated with chorioamnionitis or vaginal infection during pregnancy. ${ }^{10}$ In our protocols, the vaginal cultures are not indicated routinely in the use of cervical pessary. However, it can be collected if some pathological discharge is suspected by the clinical signs, such as characteristic odor or inflammatory signs present. In the collection of culture material, it is necessary to avoid the use of speculum exam and must prioritize the collection with vaginal swabs, to not change the pessary position. If cultures are positive for pathological condition as Bacterial vaginosis (Ureaplasma, Mycoplasma, Gardnerella vaginalis, Mobiluncus, Trichomonas, Chlamydia tracomatis, Neisseria gonorrhea or other anaerobic bacteria) the treatment must be indicated with the appropriated antibiotic.

\section{How is it possible to differentiate large discharge from PPROM?}

Regularly, the CP user can present a large volume of the discharge ${ }^{10}$ probably because of the association between $\mathrm{CP}$, progesterone, and the space under the pessary, causing difficult differentiation of PPROM. The fluid is very similar to the amniotic fluid at the term of gestation with a lot of caseous vernix. However, the CP is used for patients with risk of preterm delivery, during the preterm period. During the middle gestation and 37 weeks, vernix caseosa is very rare in the amniotic fluid. ${ }^{38}$ Consequently, if a vernix caseosa-like condition could be observed during the preterm period, probably PPROM will not have occurred. If laboratory tests are not available for detection of PPROM, the combination of these clinical aspects of discharge/ amniotic fluid and a value $\geq 10$ from Amniotic Fluid Index obtained by Obstetric Ultrasound has a high negative predictive value (over 96\%) for PPROM. ${ }^{39}$

\section{Which is the best form to apply vaginal progesterone inside the vagina with a CP to avoid discharge?}

Some practice orientation can reduce discharge. Cervical pessary users should not put the capsule of progesterone close to the vaginal posterior fornix and should put the capsule in the distal third of the vagina, immediately before sleep. In the morning, during her first urine, the patient needs to open the vaginal lips with her fingers, making a little push to eliminate accumulated fluid, which can avoid a lot of volume of discharge during the day.

\section{Clinical aspects}

\section{What is the best treatment for small pain during the use of a pessary?}

Analgesics can be helpful in those particular cases, but some patients have nerve compression after $\mathrm{CP}$ insertion and this neural pain is not responsive to drugs. Our suggestion is to change the position during sleep or rest because the lateral decubitus of the patient makes the pessary reduce the compression of the pelvic nerves.

\section{What is the best treatment for obstipation if it occurs?}

The obstipation can follow CP insertion. We start the orientation by increasing fiber in the diet, suggesting the ingestion of fruits, vegetables, yogurt, and hydration. If obstipation continues, the best medication to start the treatment is glycerin suppository and finalizes, if necessary, a $100-\mathrm{mL}$ enema.

How long is it possible to have urinary symptoms after pessary insertion and do these symptoms has any association with urinary infection?

Frequently, after CP insertion, the pregnant women complain about urinary symptoms, as dysuria, urgency, or vesical small pain, which disappear after 48 hours, without any medication use. In our experience, there is no association with urinary infection.

\section{Is the rotation of the CP around the cervix a good} practice for all types of pessaries available?

In Brazil, a similar Arabin pessary is frequently used, but the small orifice has an irregular internal diameter, producing a kind of "grab" in the cervix, and the rotation is not recommended (Figure 2). In Arabin pessary, the rotation can be useful for the adjustment of CP position after insertion.

\section{After inhibition of labor, is the outcome similar to the patient without the device, with regular subsequent delivery? Or the inhibition with CP can increase the time of pregnancy?}

If the patient presents preterm labor after $\mathrm{CP}$ insertion, it must be considered inhibition; for a singleton pregnancy, the best treatment is Atosiban, ${ }^{40}$ and for a twin pregnancy, the best treatment is Nifedipine. ${ }^{41}$ Although the inhibition is preconized only for stopping palliatively the labor to corticosteroids application, during $\mathrm{CP}$ treatment the inhibition can cause a permanent change and, after the period of using the drug for inhibition, sometimes labor does not occur anymore, as well as the 
delivery. There is a lack of evidence about this issue and new studies with a cervical pessary and preterm labor have been conceived to check this aspect. Actually, because of CP placement, the diagnosis of preterm labor is exclusively by abdominal contraction, thus the cervix is always closed by pessary, and more comprehension about the physiopathology of the labor with pessary is necessary.

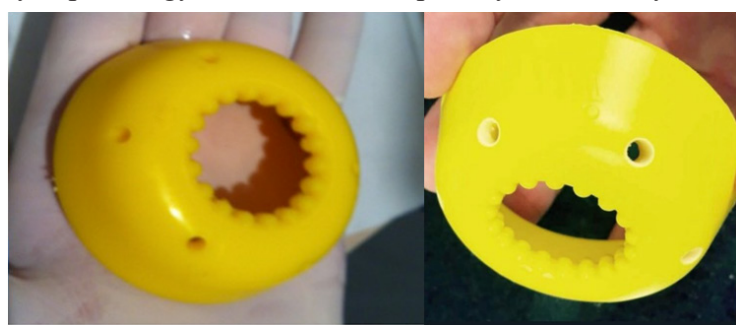

Figure $2 \mathrm{~A}$ single-size Brazilian CP usually inserted, similar to Arabin pessary, with an irregular internal orifice, with measures of $60 \times 20 \times 27 \mathrm{~mm}$ (major diameter $\mathrm{x}$ height $\mathrm{x}$ minor diameter).

\section{How important is information about the method for pessary removal, considering stuck pessary?}

Pessary removal is indicated during $37^{\text {th }}$ week gestation and can be painful, but if the responsible doctor is concerned about cervical lesions during extraction, it will probably not occur. Soft movements are necessary to remove the $\mathrm{CP}$. Sometimes pessary can be immovable inside the vagina, in this case, some maneuvers are necessary, such as pushing the cervix inside the small orifice of $\mathrm{CP}$, combined with soft traction of the CP to the distal vagina, and it well succeeded in the majority of the cases. In our casuistry, all cases were removed with this technique, without any cervix lesion. Another possibility to withdraw $\mathrm{CP}$ is cutting the fixed device with a curved-bladed Mayo scissors.

\section{What are the medical aspects to consider for the removal of the device $<34$ weeks?}

The withdrawal must be considered after PPROM and preterm labor without response inhibition. After PPROM $<34$ weeks without clinical and laboratory signs of infection, the maintenance of $\mathrm{CP}$ in place for 48 hours could be considered, after hospital admission, for antenatal corticosteroids. ${ }^{42}$

\section{Conclusion}

The most important aspect related to the $\mathrm{CP}$ is the experience. The studies with this device are more complex than studies containing only medication. Therefore, if the author does not have the experience needed, their results could have bias. New guidelines must be developed to orient new users or new trials in CP. There is a lack of evidence in some aspects about $\mathrm{CP}$, such as inhibition with $\mathrm{CP}$, obstipation, treatment of pelvic pain, urinary symptoms, and the security of maintenance of CP in place after PPROM.

\section{Acknowledgments}

The authors acknowledge Mr. Rudolf Wiedemann and Ms. Gabriela Ubeda with the English version of this article.

\section{Funding}

None.

\section{Conflicts of interest}

The author declares that there is no conflict of interest regarding this study.

\section{References}

1. Nicolaides KH, Syngelaki A, Poon LC, et al. A randomized trial of a cervical pessary to prevent preterm singleton birth. Obstet Gynecol Surv. 2016;71(7):392-393.

2. Saccone G, Maruotti GM, Giudicepietro A, et al. Effect of cervica pessary on spontaneous preterm birth in women with singleton pregnancies and short cervical length a randomized clinical trial. JAMA. 2017;318(23):2317-2324.

3. Goya M, Pratcorona L, Merced C, et al. Cervical pessary in pregnant women with a short cervix (PECEP): an open-label randomised controlled trial. Lancet. 2012;379(9828):1800-1806.

4. Hui SYA, Chor CM, Lau TK, et al. Cerclage pessary for preventing preterm birth in women with a singleton pregnancy and a short cervix at 20 to 24 weeks: A randomized controlled trial. Am J Perinatol. 2013;30(04):283288 .

5. Ting YH, Lao TT, Wa Law L, et al. Arabin cerclage pessary in the management of cervical insufficiency. $J$ Matern Neonatal Med. 2012;25(12):2693-2695.

6. Dugoff L, Berghella V, Sehdev H, et al. Cervical pessaries for the prevention of preterm birth: a systematic review and meta-analysis. $J$ Matern Neonatal Med. 2017;32(12):1654-1663.

7. Jin XH, Li D, Huang LL. Cervical pessary for prevention of preterm birth: a meta-analysis. J Matern Neonatal Med. 2017;7:42560.

8. Jin Z, Chen L, Qiao D, et al. Cervical pessary for preventing preterm birth: a meta-analysis. J Matern Neonatal Med. 2019;32(7):1148-1154.

9. Saccone G, Ciardulli A, Xodo S, et al. Cervical pessary for preventing preterm birth in singleton pregnancies with short cervical length: a systematic review and meta-analysis. J Matern Neonatal Med. 2017;32(1):1654-1663.

10. Conde-agudelo A, Romero R, Nicolaides KH. Cervical pessary to prevent preterm birth in asymptomatic high-risk women: a systematic review and meta-analysis. Am J Obstet Gynecol. 2020;223(1):42-65.e2.

11. Robert L Goldenberg, Jennifer F Culhane, Jay D Iams RR. Epidemiology and causes of preterm birth. Lancet. 2008;371(9606):75-84

12. Pacagnella RC, Santucci Franca M, Cecatti JG, et al. A randomized controlled trial on the use of pessary plus progesterone to prevent preterm birth in women with short cervical length (P5 trial). BMC Pregnancy Childbirth. 2019;19:442.

13. Pacagnella RC, Silva T, Cecatti JG, et al. 2 Pessary plus progesterone to prevent preterm birth in women with a short cervix (P5 trial). Am J Obstet Gynecol. 2021;224(2):S1-S2.

14. Petrou $\mathrm{S}$. The economic consequences of preterm birth during the first 10 years of life. BJOG An Int J Obstet Gynaecol. 2005;112(SUPPL. 1):10-15.

15. Russell RB, Green NS, Steiner CA, et al. Cost of hospitalization for preterm and low birth weight infants in the United States. Pediatrics. 2007;120(1).

16. Arabin B, Alfirevic Z. Cervical pessaries for prevention of spontaneous preterm birth: Past, present and future. Ultrasound Obstet Gynecol. 2013;42(4):390-399.

17. Di Renzo GC, Cabero Roura L, Facchinetti F, et al. Preterm labor and birth management: recommendations from the European association of perinatal medicine. J Matern Neonatal Med. 2017;30(17):2011-2030.

18. Kyvernitakis I, Maul H, Rath W, et al. Position paper of the task force for obstetrics and prenatal medicine (AGG - Section Preterm Birth) on the placement, removal and surveillance of the arabin cervical pessary in patients at risk for spontaneous preterm birth. Geburtshilfe Frauenheilkd. 2019;79(11):1171-1175.

19. Mattar R, Lobo G do R, Nomura RMY, et al. Conduct Protocols - Obstetrics Department - UNIFESP/EPM. 
20. McIntosh J, Feltovich H, Berghella V, et al. The role of routine cervical length screening in selected high- and low-risk women for preterm birth prevention. Am J Obstet Gynecol. 2016;215(3).

21. ACOG Practice Bulletin \#56: Multiple gestation: complicated twin, triplet, and high-order multifetal pregnancy. Obstet Gynecol. 2004;104(4):869883.

22. Brizot ML, Francisco RPV, Liao AW, et al. Vaginal progesterone for the prevention of preterm birth in twin gestations: a randomized placebocontrolled double-blind study. Am J Obstet Gynecol. 2015;213(1):82. e1-82.e9.

23. Liem S, Schuit E, Hegeman M, et al. Cervical pessaries for prevention of preterm birth in women with a multiple pregnancy (ProTWIN): A multicentre, open-label randomised controlled trial. Lancet. 2013;382(9901):1341-1349.

24. Goya M, De La Calle M, Pratcorona L, et al. Cervical pessary to prevent preterm birth in women with twin gestation and sonographic short cervix: A multicenter randomized controlled trial (PECEP-Twins). Am J Obstet Gynecol. 2016;214(2):145-152.

25. Liem SMS, van Pampus MG, Mol BWJ, et al. Cervical pessaries for the prevention of preterm birth: a systematic review. Obstet Gynecol Int. 2013.

26. Nicolaides KH, Syngelaki A, Poon LC, et al. Cervical pessary placement for prevention of preterm birth in unselected twin pregnancies: A randomized controlled trial. Am J Obstet Gynecol. 2016;214(1):3.e1-3.e9.

27. Franca MS, Hatanaka AR, Andrade Jr VL, et al. Cervical pessary plus progesterone for twin pregnancy with short cervix compared to unselected and non-treated twin pregnancy: A historical equivalence cohort study (EPM Twin Pessary Study). Rev Bras Ginecol e Obs. 2020;42(10):621629.

28. ACOG Practice Bulletin \# 142 Cerclage for the management of cervical insufficiency. Obstet Gynecol. 2014;123(2):372-379.

29. Romero R, Yeo L, Miranda J, et al. A blueprint for the prevention of preterm birth: vaginal progesterone in women with a short cervix Roberto. J Perinat Med. 2013;41(1):27-44.

30. Hatanaka AR, Franca MS, Hamamoto TENK, et al. Antibiotic treatment for patients with amniotic fluid "sludge" to prevent spontaneous preterm birth: A historically controlled observational study. Acta Obstet Gynecol Scand. 2019:1157-1163.
31. Witkin SS, Moron AF, Ridenhour BJ, et al. Vaginal biomarkers that predict cervical length and dominant bacteria in the vaginal microbiomes of pregnant women. M Bio. 2019;10(1):e02242-19.

32. Jarde A, Lewis-Mikhael AM, Moayyedi P, et al. Pregnancy outcomes in women taking probiotics or prebiotics: A systematic review and metaanalysis. BMC Pregnancy Childbirth. 2018;18(1):1-14.

33. Jin WH, Ha Kim Y, Kim JW, et al. Antibiotic treatment of amniotic fluid "sludge" in patients during the second or third trimester with uterine contraction. Int J Gynecol Obstet. 2021;153(1):119-124.

34. Ples L, Sima R, Ricu A, et al. The efficacy of cervical cerclage combined with a pessary for the prevention of spontaneous preterm birth. J Matern Neonatal Med. 2019;21:1-5.

35. Barbone A, Li X, Arabin B, et al. Preliminary modeling of effective positioning of Arabin cerclage pessary in women at high risk of preterm birth. Ultrasound Obs Gyneco. 2020;55(4):557-558.

36. Goya M, Pratcorona L, Higueras T, et al. Sonographic cervical length measurement in pregnant women with a cervical pessary. Ultrasound Obstet Gynecol. 2011;38(2):205-209.

37. Mendoza M, Goya M, Gascón A, et al. Modification of cervical length after cervical pessary insertion: correlation weeks of gestation. J Matern Neonatal Med. 2017;30(13):1596-1601.

38. Nishijima K, Yoneda M, Hirai T, et al. Biology of the vernix caseosa: A review. J Obstet Gynaecol Res. 2019;45(11):2145-2149.

39. Weissmann-Brenner A, O'Reilly-Green C, Ferber A, et al. Values of amniotic fluid index in cases of preterm premature rupture of membranes. $J$ Perinat Med. 2009;37(3):232-235.

40. Verdurmen KMJ, Hulsenboom ADJ, van Laar JOEH, et al. Effect of tocolytic drugs on fetal heart rate variability: a systematic review. J Matern Fetal Neonatal Med. 2017;30(20):2387-2394.

41. Santucci Franca M, ENK Hamamoto T, Fernandes Moron A. Preterm birth in twins. Multiple Pregnancy - New Challenges; 2019.

42. ACOG Practice Bulletin \# 159 Management of preterm labor. Obstet Gynecol. 2016;127(1):e29-e38. 